\title{
Investigación, publicación e impactos deseables. ¿Cómo no morir en el intento?
}

Research, publication, and desirable impact. How not to die trying?

\author{
César Cabezas ${ }^{1, a}$ \\ ${ }^{1}$ Facultad de Medicina, Universidad Nacional Mayor de San Marcos. Lima, Perú. \\ ${ }^{a}$ Director, Anales de la Facultad de Medicina
}

\section{Correspondencia: \\ César Cabezas \\ ccabezass@unmsm.edu.pe}

Recibido: 12 de diciembre 2018

Aprobado: 20 de diciembre 2018

Conflictos de interés: Ninguno.

Citar como: Cabezas C. Investigación, publicación e impactos deseables. ¿Cómo no morir en el intento?. An Fac med. 2018;79(4):280-1.

DOI: http://dx.doi.org/10.15381/anales. v79i4.15629
Se considera que el principal objetivo de la investigación en salud es contribuir a mejorar el bienestar y las condiciones de vida de las personas, pero lo que vemos en la realidad es que el desempeño de los investigadores suele medirse por la calidad científica de sus investigaciones, más que por su impacto en la salud. Esto es marcadamente insuficiente, por lo que hace buen tiempo se está tratando de concebir una forma de medir el impacto social de la investigación en salud aplicada ${ }^{(1)}$.

Idealmente todas las investigaciones debieran ser de alta calidad y tener un impacto social considerable en el mejoramiento de la salud; sin embargo, fácticamente la calidad científica y el impacto social no siempre caminan juntos. La publicación científica se encuentra en medio de ese proceso que debe ir de la investigación a la utilidad de la evidencia.

Anales de la Facultad de Medicina desde sus inicios en 1918, así como otras revistas médicas en otras latitudes han priorizado la difusión de investigaciones, revisiones y comentarios de actualidad relacionadas a la medicina y la salud; en nuestro caso sobre temas inherentes a los problemas sanitarios del país. Es probable que en aquellos tiempos aurorales, las revistas médicas se leyeron en casi su totalidad y ese conocimiento probablemente tuvo alguna aplicación práctica en su momento. Actualmente, aún el acceso a todas la publicaciones y más aún su cabal lectura sería imposible, considerando que en las últimas décadas se ha producido una explosión de publicaciones científicas. Según el Scimago Journal Ranking -que evalúa instituciones de investigación a través de indicadores como el de publicaciones (Scopus), innovación (PATSTAT) y visibilidad en la web (google)- para el periodo 1996-2017 en el mundo se han generado 48,969,648 publicaciones científicas en el área de la medicina, de la cuales 3,704,587 (5,6\%) pertenecieron a los EEUU, país con el may- or aporte en publicaciones, en el mundo; mientras que Brasil aportó con 260,680 $(0,53 \%)$ publicaciones, siendo el primero en Sudamérica, y en el caso del Perú su aporte fue de 8,449 (0.01\%) publicaciones, ubicándose en el puesto 64 en el mundo y el puesto 13 en Latinoamérica ${ }^{(2)}$.

A través de la historia existieron tres procesos que dieron el carácter internacional de la actividad científica; el primero fue el de la circulación de personas, textos y objetos, luego el modo de producción de conocimiento $y$, finalmente, el financiamiento de la investigación. Pero fue el sistema de publicaciones el medio más eficiente de universalización de un estilo legítimo de producción, a medida que las revistas se convertían en el eje de rotación del sistema académico mundial, de modo que los indicadores bibliométricos sirvieron como fuente principal para las comparaciones internacionales ${ }^{(3)}$. Estas mediciones también sustentan los rankings de un reducido grupo de entidades que desarrollan investigación, de sus principales revistas y editoriales muchas veces oligopólicas, volviendo cada vez más periférica a la ciencia desarrollada en los espacios alejados del circuito dominante; por tal razón, Beigel señala la necesidad de crear nuevas herramientas de medición de la producción científica de la periferia, que contemplen las interacciones de sus universidades y centros de investigación en sus distintas direcciones, contextos y realidades ${ }^{(4)}$.

Aunque hay críticas a su validez y los sesgos que presenta, el factor de impacto (FI) constituye una medida de amplia cobertura sustentada en información pública, que permite establecer comparaciones que resultan de utilidad para la toma de decisiones sobre el ámbito de la actividad científica. También está vigente lo señalado por Birnbaum 
«Si no podemos medir lo que es valioso, acabaremos valorando nada más lo que es medible» ${ }^{(5)}$. Por otro lado han surgido alternativas de medición como el Altmetrics asi como la medición del impacto en redes sociales para identificar la evidencia cualitativa del impacto social real o potencial de la investigación compartida en redes sociales, específicamente en Twitter y Facebook ${ }^{(6)}$; sin embargo estos datos son muy transitorios, no tienen interpretaciones claras, siendo necesario un marco teórico de aceptación general.

La aparición del internet y las tecnologías digitales en general, así como su uso generalizado permite un mayor acceso a las publicaciones científicas; pero también se van generado otras preocupaciones, como son el real acceso a revistas indizadas, la reproducibilidad de las investigaciones publicadas, la identificación de las tendencias de la actualidad y si realmente hay un impacto en la innovación tecnológica que contribuyan en lo social. En ese contexto, podemos mencionar que han empezado a surgir como objetivos de las comunidades de investigación, en los círculos políticos y también en las entidades de financiación, lo relacionado a la ciencia abierta y la reproducibilidad de las investigaciones. Respecto a esta última, debemos destacar una publicación de Nature donde se consigna que más del $70 \%$ de los investigadores han intentado y fracasado en reproducir los experimentos de otro científico, y más de la mitad no han podido reproducir sus propios experimentos, encuesta que incluyó a 1576 investigadores ${ }^{(7)}$. Sobre la ciencia abierta es pertinente comentar el acuerdo Open Data donde se establecen los principios para maximizar el beneficio de la gran cantidad de los datos digitales en la configuración del futuro de la ciencia ${ }^{(8)}$.

Finalmente debemos mencionar que el crecimiento de la investigación científica durante las últimas décadas ha superado largamente la disponibilidad de los recursos públicos para poder financiarla, generando un problema para las agencias gubernamentales que promocionan la investigación y para los políticos, que tienen que ver cómo los recursos limitados pueden ser distribuidos de manera más eficiente y efectiva entre los investigadores y los proyectos de investigación. Este reto conlleva a la necesidad de evaluar la calidad de la investigación científica en sí misma y cómo determinar el impacto social de las mismas. Para lo primero como comentamos antes hay un conjunto de medidas que se aplican, sin embargo, ha sido mucho más difícil desarrollar medidas confiables y significativas para evaluar el impacto social de la investigación ${ }^{(9)}$. Es evidente que no existe un vínculo directo entre la calidad científica de un proyecto de investigación y su valor social y como lo señala Nightingale «las investigaciones que son altamente citadas o publicadas en las principales revistas pueden ser buenas para la disciplina académica pero no para la sociedad»(10)

Aún no existe una definición para el impacto social de la investigación. Existe conceptos diferentes, de como medir los beneficios sociales, culturales, ambientales y económicos de la investigación financiada con fondos públicos, ya sean productos o ideas. Por todo ello, se recomienda que el impacto social debe medirse de manera cuantificable, y los paneles de expertos deben revisar la evidencia narrativa en estudios de casos respaldados por indicadores apropiados. Si bien este método es una actividad casi artesanal en lugar de cuantitativa, parece ser la mejor manera de medir el complejo fenómeno del impacto social ${ }^{(9)}$.

Como señalamos, el impacto social es mucho más difícil de medir que el impacto científico, y probablemente no existan indicadores que puedan utilizarse en todas las disciplinas e instituciones para la recopilación en las bases de datos. Asi mismo puede tomar muchos años para que ese impacto se haga evidente. La construcción y validación de estos indicadores debe resultar de una interacción entre investigadores, evaluadores, decisores y potenciales beneficiarios. Como decíamos al inicio de este editorial, si el principal objetivo de la investigación en salud es contribuir a mejorar el bienestar y las condiciones de vida de las personas, la calidad de la investigación científica y su impacto social, a la vez de ser un reto, debe ser un deber moral para todos los que coincidimos en buscar dichos objetivos y no morir en el intento.

\section{REFERENCIAS BIBLIOGRÁFICAS}

1. Smith R. Measuring the social impact of research. BMJ. 2001;323(7312):528.

2. Scimago Journal \& Country Rank [Internet]. Michigan: Scimago Lab; c2007-2018 [citado el 12 de diciembre de 2018]. Disponible en: https://www.scimagojr.com/ countryrank.php?area=2700

3. Gingras $Y$. Les formes spécifiques de l'internationalité du champ scientifique. Actes de la Recherche en Sciences Sociales. 2002;(141-142):31-45.

4. Beigel F. Las relaciones de poder en la ciencia mundial. Un anti-ranking para conocer la ciencia producida en la periferia. Nueva Sociedad. 2018;(274):13-28.

5. Gil MA. Réplica a un siglo buscando doctores... iy ya los encontramos! Revista de la Educación Superior. 2006;35(4):129-140.

6. Pulido CM, Redondo-Sama G, SordeÂ-MartõÂ T, Flecha R. Social impact in social media: A new method to evaluate the social impact of research. PLoS One. 2018;13(8):e0203117.

7. Baker M. 1,500 scientists lift the lid on reproducibility. Nature. 2016;533(7604):452-4.

8. Boulton G. Reproducibility: International accord on open data. Nature. 2016;530(7590):281.

9. Bornmann L. Measuring the societal impact of research: Research is less and less assessed on scientific impact alone-we should aim to quantify the increasingly important contributions of science to society. EMBO Rep. 2012;13(8):673-6.

10. Nightingale $P$, Scott A. Peer review and the relevance gap: ten suggestions for policy-makers. Sci Public Policy. 2007;34(8):543-53. 